\title{
BMJ Open Visual dysfunction and its correlation with retinal changes in patients with Parkinson's disease: an observational cross-sectional study
}

\author{
V Polo, ${ }^{1,2}$ M Satue, ${ }^{1,2}$ M J Rodrigo, ${ }^{1}$ S Otin, ${ }^{1,2}$ R Alarcia, ${ }^{1,3}$ M P Bambo, ${ }^{1,2}$ \\ M I Fuertes, ${ }^{1,2}$ J M Larrosa, ${ }^{1,2}$ L E Pablo, ${ }^{1,2}$ E Garcia-Martin ${ }^{1,2}$
}

To cite: Polo V, Satue M, Rodrigo MJ, et al. Visual dysfunction and its correlation with retinal changes in patients with Parkinson's disease: an observational cross-sectional study. BMJ Open 2016;6: e009658. doi:10.1136/ bmjopen-2015-009658

- Prepublication history for this paper is available online. To view these files please visit the journal online (http://dx.doi.org/10.1136/ bmjopen-2015-009658).

Study design and setting: an observational cross-sectional study, carried out at Miguel Servet University Hospital, Zaragoza, Spain

Received 6 August 2015 Revised 3 March 2016 Accepted 11 April 2016

${ }^{1}$ IIS Aragon, Institute for Health Sciences of Aragon, Zaragoza, Spain

20phthalmology Department, Miguel Servet University Hospital, Zaragoza, Spain ${ }^{3}$ Neurology Department, Miguel Servet University Hospital, Zaragoza, Spain

Correspondence to Dr M Satue;

mariasatue@gmail.com

\section{ABSTRACT}

Objectives: To evaluate visual dysfunction and its correlation with structural changes in the retina in patients with Parkinson's disease (PD).

Methods: Patients with PD $(n=37)$ and controls $(\mathrm{n}=37)$ were included in an observational crosssectional study, and underwent visual acuity (VA), colour vision (using the Farnsworth and Lanthony desaturated D15 colour tests) and contrast sensitivity vision (CSV; using the Pelli-Robson chart and CSV 1000 E test) evaluation to measure visual dysfunction. Structural measurements of the retinal nerve fibre layer (RNFL), and macular and ganglion cell layer (GCL) thicknesses, were obtained using spectral domain optical coherence tomography (SD-OCT). Comparison of obtained data, and correlation analysis between functional and structural results were performed.

Results: VA (in all different contrast levels) and all CSV spatial frequencies were significantly worse in patients with PD than in controls. Colour vision was significantly affected based on the Lanthony colour test. Significant GCL loss was observed in the minimum GCL+inner plexiform layer. A clear tendency towards a reduction in several macular sectors (central, outer inferior, outer temporal and superior (inner and outer)) and in the temporal quadrant of the RNFL thickness was observed, although the difference was not significant. CSV was the functional parameter most strongly correlated with structural measurements in PD. Colour vision was associated with most GCL measurements. Macular thickness was strongly correlated with macular volume and functional parameters $(r>0.70, p<0.05)$.

Conclusions: Patients with PD had visual dysfunction that correlated with structural changes evaluated by SD-OCT. GCL measurements may be reliable indicators of visual impairment in patients with PD.

\section{INTRODUCTION}

Foveal vision alterations are associated with Parkinson's disease (PD), and seem to be caused by dysfunction of the intraretinal dopaminergic circuitry and final retinal

\section{Strengths and limitations of this study}

- This study includes a complete assessment of visual function parameters and the evaluation of different retinal structures using spectral domain optical coherence tomography in patients with Parkinson's disease.

- There are only two other published articles evaluating the association between visual dysfunction and morphological parameters. Results provided by these previous studies differ from our results, possibly due to different measurement methods and sample size.

- Colour vision in our study was assessed by Lanthony and Farnsworth D15 colour tests, which may provide more specific information about colour deficiencies. These tests are not commonly used to evaluate colour deficiencies in patients with $\mathrm{PD}$.

- An important limitation to our study is the inclusion of one randomly selected eye per patient. The incorporation of both eyes of each patient in Parkinson's disease studies is usually recommended due to asymmetrical involvement of the retina in this process.

output to the brain. ${ }^{1}$ Recent studies demonstrated retinal thinning in patients with PD compared with healthy participants. ${ }^{2-5}$

Several studies report a correlation between functional disability and axonal loss observed in the optic nerve in multiple sclerosis, another neurodegenerative process. ${ }^{6} 7$ Patient with PD are also reported to have decreased contrast sensitivity and colour vision, and altered visual evoked potentials. ${ }^{1}{ }^{8-13}$ To our knowledge, however, very few studies have assessed visual dysfunction in PD and its correlation with morphological parameters. ${ }^{14} 15$

In the present study, we evaluated visual acuity (VA), using an Early Treatment Diabetic Retinopathy Study (ETDRS) chart, 
contrast sensitivity vision (CSV), using the CSV-1000E test and Pelli-Robson chart, and colour vision, using the Farnsworth and Lanthony tests in patients with PD and healthy controls, to examine the association between visual dysfunction and morphological parameters.

\section{MATERIALS AND METHODS}

Thirty-seven eyes of 37 patients with definite PD and 37 eyes of 37 age-matched and sex-matched healthy individuals were recruited for an observational cross-sectional study. The study was performed at Miguel Servet University Hospital in Zaragoza, Spain, and all evaluations were performed in one single visit. All procedures adhered to the tenets of the Declaration of Helsinki, and all participants provided informed consent to participate in the study.

The diagnosis of PD was based on standard clinical and neuroimaging criteria. ${ }^{16}$ Information about disease severity was assessed using the Hoehn-Yahr scale ${ }^{17}$ and the Unified Parkinson Disease Rating Scale part III score (UPDRS III) ${ }^{18}$ Disease duration and treatment were recorded. Exclusion criteria were the presence of significant refractive errors ( $>5$ dioptres of spherical equivalent refraction or 3 dioptres of astigmatism); intraocular pressure $\geq 21 \mathrm{~mm} \mathrm{Hg}$; media opacification; concomitant ocular diseases, including history of glaucoma or retinal pathology; and systemic conditions that could affect the visual system. The healthy controls had no history and no evidence of ocular or neurological disease of any nature; their best-corrected VA (BCVA) was $>20 / 30$ based on the Snellen scale.

All participants underwent a complete neuro-ophthalmic evaluation, which included pupillary, anterior segment and funduscopic examination. Visual function was assessed by evaluating BCVA, using an ETDRS chart, CSV, using the CVS-1000E test and Pelli-Robson chart, and colour vision, using the Farnsworth desaturated D15 and Lanthony desaturated D15 tests. Structural analysis of the retina was performed using spectral domain (SD) optical coherence tomography (OCT) with a Cirrus high definition OCT (Carl Zeiss Meditec Inc, Dublin, California, USA), which included three different protocols: macular protocol (for macular thickness analysis), retinal nerve fibre layer (RNFL) protocol and ganglion cell protocol (for individual analysis of this layer).

LogMAR VA was evaluated at three different contrast levels: $100 \%$ (HCVA, using ETDRS chart), $2.50 \%$ and $1.25 \%$ (LCVA, using Low-Contrast Sloan Letter Charts, Precision Vision, LaSalle, Illinois, USA), with the percentage indicating the level of contrast, that is, $100 \%$ representing black letters over white background and $1.25 \%$ representing light grey letters over white background. All measurements were obtained under monocular vision and controlled lighting conditions with best correction.

Contrast sensitivity provides more complete information about visual function than do VA tests. CSV was evaluated in our patients, using the Pelli-Robson chart and the CVS-1000E test. The Pelli-Robson chart comprises horizontal lines of capital letters organised into groups of three (triplets), with two triplets per line. Within each triplet, all letters have the same contrast. The contrast decreases from one triplet to the next, even within each line. All patients were evaluated under both monocular and binocular vision at a distance of $1 \mathrm{~m}$ from the chart, and under controlled fotopic conditions $\left(85 \mathrm{~cd} / \mathrm{m}^{2}\right)$. The score corresponding to the last triplet of letters seen by the patient was recorded.

The CSV-1000E instrument is used worldwide for standardised CSV and glare testing. All patients were evaluated at a distance of $2.5 \mathrm{~m}$ from the chart, under monocular vision, at four different spatial frequencies $(3,6,12$ and 18 cycles per degree (cpd)). The chart comprises four rows with 17 circular patches each. The patches present a grating that decreases in contrast, moving from left to right across the row. The patient indicates whether the grating appears in the top patch or the bottom patch for each column. Each contrast value for each spatial frequency was transformed into a logarithmic scale according to standardised values.

Colour vision was assessed using the Color Vision Recorder (CVR) program. CVR software analyses chromatic discrimination by classification of colours. The program includes the classic test of Farnsworth 100-hue (FM-100), Farnsworth-Munsell D15 and Lanthony D15. All patients in the study were evaluated using the Farnsworth-Munsell D15 and Lanthony D15 protocols, and different output parameters such as the confusion index (C-index), the colour confusion index (CCI), the confusion angle (Conf Ang) and the scatter index (S-index) were recorded. ${ }^{19} 20$ The tests were performed under monocular vision.

Structural measurements of the retina were obtained using the Cirrus OCT device. The same experienced operator performed all scans and did not apply manual correction to the OCT output. We used an internal fixation target because it provides the highest reproducibility and rejected poor quality scans prior to data analysis. The Cirrus OCT macular cube $512 \times 128$ protocol provides a macular volume measure and retinal thickness values for nine areas. These areas include a central $1 \mathrm{~mm}$ circle representing the fovea, and inner and outer rings measuring 3 and $6 \mathrm{~mm}$ in diameter, respectively. The inner and outer rings are divided into four quadrants each. The Cirrus OCT optic disc protocol generates images with 200 linear scans enabling analysis of the RNFL of a $6 \mathrm{~mm}^{3}$ area around the optic nerve. For each scan series of RNFL measurements, we assessed the average, superior, inferior, temporal and nasal thickness. Cirrus segmentation analysis for retinal layers also provides measurements of the ganglion cell layer (GCL) thickness, evaluating six areas of the macular cube (superior, superonasal, inferonasal, inferior, inferotemporal and superotemporal sectors), and measurements of the average and minimum GCL plus the inner 
Table 1 Epidemiological and disease characteristics of patients with PD and healthy participants, and statistical significance ( $p$ )

\begin{tabular}{|c|c|c|c|}
\hline Parameter & Controls & Parkinson disease & p Value \\
\hline Number of eyes (n) & 37 & 37 & - \\
\hline Age, years, range & $68(60-76)$ & $69(58-74)$ & 0.361 \\
\hline Men:women (\% of men) & $24: 13(64.9)$ & $23: 14(62.2)$ & 0.441 \\
\hline Intraocular pressure & $15.58(2.71)$ & $15.12(2.98)$ & 0.720 \\
\hline Disease duration, years, mean (SD) & - & $13.2(5.77)$ & - \\
\hline Hoehn-Yahr, mean (SD) & - & $2.7(0.64)$ & - \\
\hline UPDRS III, mean (SD) & - & $25.06(8.24)$ & - \\
\hline
\end{tabular}

plexiform layer (GCL+IPL) value of a set of 360 spokes, where each average represents the mean number of the pixels along the spoke that lies within the measurement annulus. The minimum is selected because the thinnest portion of the GCL+IPL in the perifoveal region is considered to indicate damage to the ganglion cells.

All data analyses were performed using SPSS software V.20.0 (SPSS Inc, Chicago, Illinois, USA). Owing to the parametric distribution of the data, differences between evaluations of patients with PD and healthy participants were compared using Student's t test. To avoid a high false-positive rate, the Bonferroni correction for multiple comparisons was calculated. The level of significance for each variable was established based on Bonferroni calculations.

The linear correlation between structural and functional parameters was determined using Pearson's correlation coefficient. Values of $\mathrm{p}<0.05$ were considered to indicate a significant correlation. Each eye was considered separately, and one eye from each patient was randomly selected for analysis.

\section{RESULTS}

Thirty-seven patients with PD and 37 healthy controls were included in the study. The mean age of the patients with PD was 69 years (range 58-74 years) and the mean age of the healthy controls was 68 years (range $60-76$ years $)$. Age $(\mathrm{p}=0.361)$, sex $(\mathrm{p}=0.441)$ and intraocular pressure $(p=0.720)$ did not differ significantly between healthy controls and patients with PD. Mean time from diagnosis of PD was 13.2 years. The median Hoehn-Yahr stage was 2.7, and the stage of PD based on the UPDRS III was 25.06 (range 7-39; table 1).

Treatment was divided into three different categories: 'drugs that enhance dopamine levels' (carbidopa, levodopa and rasagiline), 'dopaminergic drugs' (pramipexole, ropirinol and rotigotine) and 'other' (amitriptiline, propranolol and clonazepam). 'Drugs that enhance dopamine levels' was the most prescribed category $(89 \%$ of patients), and combination therapy with levodopa and carbidopa was the most frequent treatment (44\%). Sixty-four per cent of treatments were categorised as 'dopaminergic', most of which were used in combination with drugs included in the previous category. A small percentage of patients $(9 \%)$ were prescribed drugs with no dopaminergic effects.

\section{Functional parameters}

Patients with PD had a lower BCVA at all three contrast levels of the ETDRS chart compared with the controls $(0.18 \pm 0.26$ in patients vs $-0.065 \pm 0.9$ in controls at $100 \%$, $\mathrm{p}=0.001 ; 0.59 \pm 0.21$ vs $0.44 \pm 0.13$ at $2.50 \%, \mathrm{p}=0.010$; and $0.61 \pm 0.23$ vs $0.58 \pm 0.16$ at $1.25 \%, \mathrm{p}=0.009)$. CSV was affected in patients at all four spatial frequencies of the CSV 1000E chart (3, 6, 12 and $18 \mathrm{cpd}$ ) when analysed based on the number of correct localised gratings ( $\mathrm{p}=0.001, \mathrm{p}<0.001, \mathrm{p}<0.001$ and $\mathrm{p}=0.004$, respectively). The Pelli-Robson results also revealed a significant reduction in CSV in patients with PD (1.71 in patients vs 1.89 in controls, $\mathrm{p}=0.02$ ). Colour vision (Conf Angle in Lanthony test) was also affected in PD. The results are shown in table 2.

\section{Structural parameters}

Based on Bonferroni corrections, OCT measurements indicated a significant difference in the minimum GCL + IPL value $(80.18 \pm 6.19$ vs $82.45 \pm 3.60 \mu \mathrm{m} ; \mathrm{p}=0.005)$. However, we observed a clear tendency towards a reduction in superior macular sectors in the outer inferior, outer temporal and central macular thickness in patients with PD compared with controls: the $\mathrm{p}$ value for these variables was $<0.05$ but did not meet Bonferroni significance (results are shown in table 2). The segmentation analysis revealed a tendency towards reduced GCL in patients with $\mathrm{PD}$ in the superior $(81.64 \pm 7.08 \mu \mathrm{m}$ in patients vs $84.55 \pm 4.32 \mu \mathrm{m}$ in controls; $\mathrm{p}=0.032$ ) and superonasal sectors $(81.04 \pm 7.23$ vs $85.28 \pm 4.78 \mu \mathrm{m}$; $\mathrm{p}=0.029$ ); and the RNFL was reduced in the temporal quadrant in patients with PD (table 3). These parameters, however, did not meet the level of significance established by Bonferroni correction.

\section{Correlation between functional and structural parameters}

CSV was the functional parameter most frequently associated with structural measurements in PD. The Pelli-Robson CSV results correlated with GCL thickness in all sectors, although the association was not strong $(\mathrm{r}<0.5)$. The superonasal $(\mathrm{r}=0.40, \mathrm{p}=0.010)$, inferonasal $(\mathrm{r}=0.40, \quad \mathrm{p}=0.010), \quad$ inferior $\quad(\mathrm{r}=0.43, \mathrm{p}=0.005)$, 
Table 2 Mean and SD of visual functional parameters in healthy controls and participants with Parkinson's disease

\begin{tabular}{|c|c|c|c|c|c|}
\hline & \multicolumn{2}{|c|}{ Healthy controls } & \multicolumn{2}{|c|}{$\begin{array}{l}\text { Parkinson's disease } \\
\text { patients }\end{array}$} & \multirow[b]{2}{*}{ Significance (p) } \\
\hline & Mean & SD & Mean & SD & \\
\hline VA ETDRS 100 & -0.06 & 0.096 & 0.18 & 0.26 & $0.001^{*}$ \\
\hline VA ETDRS 2.5 & 0.44 & 0.13 & 0.59 & 0.22 & $0.010^{*}$ \\
\hline VA ETDRS 1.25 & 0.58 & 0.16 & 0.62 & 0.23 & $0.009^{*}$ \\
\hline Pelli-Robson & 1.89 & 0.11 & 1.71 & 0.17 & $0.002^{*}$ \\
\hline CSV $10003 \mathrm{cpd}$ & 1.72 & 0.16 & 1.49 & 0.35 & $0.001^{*}$ \\
\hline CSV $10006 \mathrm{cpd}$ & 1.94 & 0.13 & 1.62 & 0.34 & $<0.001^{*}$ \\
\hline CSV $100012 \mathrm{cpd}$ & 1.62 & 0.17 & 1.26 & 0.41 & $<0.001^{*}$ \\
\hline CSV $100018 \mathrm{cpd}$ & 1.11 & 0.22 & 0.73 & 0.34 & $0.004^{*}$ \\
\hline Farnsworth $\mathrm{AC} \mathrm{CCl}$ & 1.11 & 0.22 & 0.73 & 0.34 & 0.851 \\
\hline Farnsworth C-index & 1.10 & 0.20 & 1.24 & 0.42 & 0.093 \\
\hline Farnsworth CCl & 1.07 & 0.12 & 1.14 & 0.24 & 0.110 \\
\hline Farnsworth Conf Angle & 63.90 & 11.15 & 65.84 & 7.49 & 0.392 \\
\hline Farnsworth S-index & 1.56 & 0.22 & 1.64 & 0.39 & 0.278 \\
\hline Farnsworth time & 78.67 & 28.96 & 82.91 & 33.10 & 0.616 \\
\hline Lanthony $\mathrm{AC} \mathrm{CCl}$ & 1.05 & 0.19 & 1.02 & 0.18 & 0.489 \\
\hline Lanthony C-index & 1.43 & 0.39 & 1.64 & 0.53 & 0.058 \\
\hline Lanthony CCI & 1.30 & 0.23 & 1.44 & 0.37 & 0.066 \\
\hline Lanthony Conf Angle & 62.31 & 14.74 & 71.91 & 9.25 & $0.002^{*}$ \\
\hline Lanthony S-index & 1.69 & 0.43 & 1.95 & 0.48 & 0.020 \\
\hline Lanthony time & 77.14 & 25.99 & 84.09 & 39.31 & 0.431 \\
\hline
\end{tabular}

The asterisk indicates those values with statistical significance after Bonferroni correction for multiple tests ( $p<0.0125$ for VA ETDRS 100, 2.50 and 1.25; $p<0.0125$ for Pelli-Robson and CSV 1000E measurements; $p<0.0083$ for Farnsworth and Lanthony tests).

$\mathrm{AC} \mathrm{CCl}$, age-corrected colour confusion index; $\mathrm{CCl}$, colour confusion index; C-index, confusion index; Conf Angle, confusion angle; cpd, cycles per degree; ETDRS, Early Treatment Diabetic Retinopathy Study; PD, Parkinson's disease; S-index, scatter index; VA, visual acuity.

superotemporal sector $(\mathrm{r}=0.43, \mathrm{p}=0.006)$ and average GCL+IPL ( $\mathrm{r}=0.45, \mathrm{p}=0.004)$ values had the highest correlations. The Pelli-Robson results also correlated with the thickness in different sectors of the RNFL (average, superior and inferior sectors). Measurements with the CSV $1000 \mathrm{E}$ at different spatial frequencies correlated significantly with most GCL measurements. The superonasal $(\mathrm{r}=0.40, \mathrm{p}=0.013)$ and superotemporal $(\mathrm{r}=0.44$, $\mathrm{p}=0.006)$ thickness, average GCL+IPL thickness $(\mathrm{r}=0.40$, $\mathrm{p}=0.012)$ and the minimum GCL+IPL $(\mathrm{r}=0.40, \mathrm{p}=0.011)$ at a spatial frequency of $6 \mathrm{cpd}$; and the superotemporal $(\mathrm{r}=0.41, \mathrm{p}=0.01)$ thickness and the minimum GCL+IPL thickness $(\mathrm{r}=0.43, \mathrm{p}=0.006)$ at a spatial frequency of $18 \mathrm{cpd}$, had the strongest correlations between CSV $1000 \mathrm{E}$ and GCL thickness. Spatial frequencies of 6 and $18 \mathrm{cpd}$ were strongly correlated with average macular thickness $(\mathrm{r}=0.79, \mathrm{p}=0.012 ; \mathrm{r}=0.77, \mathrm{p}=0.016$, respectively) and macular volume $(\mathrm{r}=0.78, \mathrm{p}=0.013 ; \mathrm{r}=0.78, \mathrm{p}=0.014$, respectively, figure 1 ).

Colour vision assessed by the Lanthony test was also associated with the structural parameters: the C-index and CCI results were significantly correlated with all outer macular parameters and most of the GCL measurements (see table 4). A significant association between colour vision and the RNFL parameters was only found in isolated sectors (see table 4).

The strongest correlation was between the average macular thickness and macular volume, and the Lanthony CCI, C-index and S-index results. No significant correlations were found between the Farnsworth's test parameters and structural measurements.

The VA ETDRS results correlated strongly with average macular thickness and macular volume (see table 5, figures 2 and 3). There were significant but mild associations between the GCL parameters and VA at $100 \%$ (superonasal, inferonasal and average GCL+IPL thickness, $\mathrm{r}=-0.38, \mathrm{p}=0.016 ; \mathrm{r}=-0.35, \mathrm{p}=0.016$; and $\mathrm{r}=0.35$, $\mathrm{p}=0.029$, respectively) and $2.50 \%$ (superonasal sector, $\mathrm{r}=$ $-0.36, \mathrm{p}=0.023$ ).

There was a significant correlation between Hoehn-Yahr score and VA contrast level 2.50\% ( $\mathrm{r}=0.48$, $\mathrm{p}=0.040$ ), and CS measured with CSV 1000 at a space frequency of $12 \mathrm{cpd}(\mathrm{r}=-0.59, \mathrm{p}=0.038)$. No correlations were detected between structural and disease severity parameters.

\section{DISCUSSION}

In the present study, we evaluated the visual function parameters, and assessed the association between visual dysfunction and morphological changes in the retina of 37 patients with PD. Parameters corresponding to VA at different contrast levels, and all CSV test results, were altered in patients with PD in comparison with healthy participants, prior to and after statistical correction for multiple tests. Moreover, contrast sensitivity was the most affected parameter in our study and correlated with most of the structural data. Colour vision was measured 
Table 3 Mean and SD of structural parameters (RNFL, GCL and macular thicknesses) obtained with the Cirrus HD optical coherence tomography device in healthy controls and participants with Parkinson's disease

\begin{tabular}{|c|c|c|c|c|c|}
\hline \multirow[b]{2}{*}{ Structural parameters } & \multicolumn{2}{|c|}{ Controls } & \multicolumn{2}{|c|}{ Parkinson's disease } & \multirow[b]{2}{*}{ p Value } \\
\hline & Mean & SD & Mean & SD & \\
\hline \multicolumn{6}{|l|}{ Macular measurements } \\
\hline Central macular thickness & 254.75 & 17.903 & 248.96 & 17.765 & 0.028 \\
\hline Inner superior macular thickness & 327.34 & 13.094 & 325.73 & 19.329 & 0.019 \\
\hline Inner nasal macular thickness & 328.52 & 13.263 & 325.45 & 17.098 & 0.091 \\
\hline Inner inferior macular thickness & 326.14 & 13.179 & 324.82 & 17.921 & 0.106 \\
\hline Inner temporal macular thickness & 315.90 & 13.615 & 312.82 & 15.760 & 0.945 \\
\hline Outer superior macular thickness & 284.76 & 9.418 & 279.44 & 17.981 & 0.008 \\
\hline Outer nasal macular thickness & 302.41 & 12.167 & 299.18 & 17.064 & 0.074 \\
\hline Outer inferior macular thickness & 277.79 & 10.755 & 273.76 & 16.798 & 0.045 \\
\hline Outer temporal macular thickness & 271.52 & 10.992 & 266.23 & 18.987 & 0.013 \\
\hline \multicolumn{6}{|l|}{ GCL thickness } \\
\hline Superior & 84.55 & 4.323 & 81.61 & 7.087 & 0.032 \\
\hline Superonasal & 85.28 & 4.780 & 81.04 & 7.234 & 0.029 \\
\hline Inferonasal & 84.66 & 5.314 & 81.82 & 7.521 & 0.135 \\
\hline Inferior & 84.34 & 5.052 & 81.91 & 6.252 & 0.389 \\
\hline Inferotemporal & 85.79 & 4.003 & 83.73 & 4.860 & 0.233 \\
\hline Temporal & 83.76 & 3.324 & 82.27 & 5.312 & 0.069 \\
\hline Average IPL+GCL & 84.83 & 4.071 & 82.73 & 6.230 & 0.095 \\
\hline Minimum IPL+GCL & 82.45 & 3.601 & 80.18 & 6.194 & $0.005^{\star}$ \\
\hline \multicolumn{6}{|l|}{ RNFL thickness } \\
\hline Average & 96.17 & 6.714 & 94.88 & 11.505 & 0.105 \\
\hline Superior & 117.90 & 10.965 & 118.68 & 16.861 & 0.115 \\
\hline Nasal & 73.59 & 12.724 & 72.40 & 15.182 & 0.345 \\
\hline Inferior & 128.14 & 14.060 & 123.20 & 22.907 & 0.075 \\
\hline Temporal & 64.97 & 8.218 & 61.48 & 10.553 & 0.027 \\
\hline
\end{tabular}

using two different tests, the Farnsworth and Lanthony $15 \mathrm{D}$ tests. These tests provide information for differentiating participants with severe loss of colour vision from

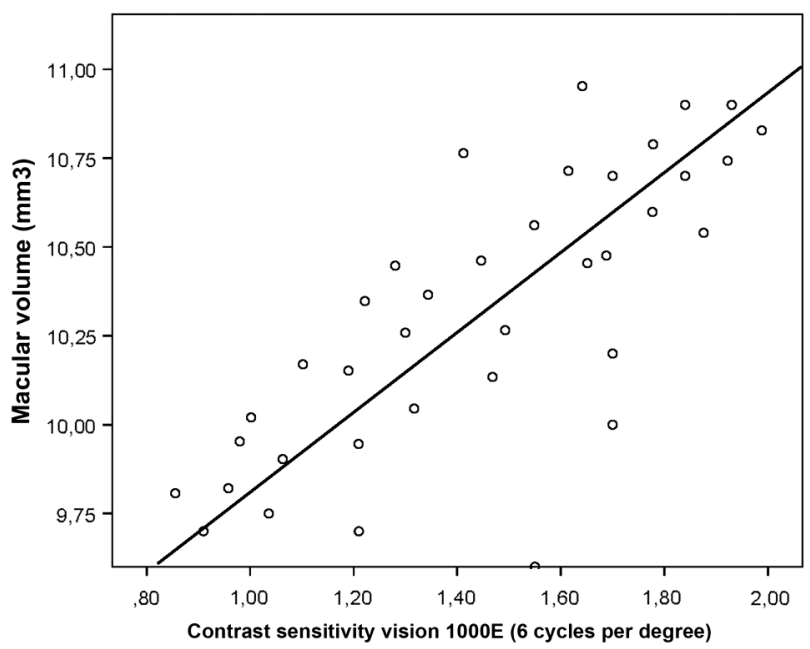

Figure 1 Correlation between the average macular thickness and contrast sensitivity vision as measured with the CSV $1000 \mathrm{E}$ test, at a spatial frequency of 6 cycles per degree, in patients with Parkinson's disease. CSV, contrast sensitivity vision. those with milder colour defects or normal colour vision, and also can be used to evaluate acquired loss of colour vision. In our study, only the Lanthony Conf Ang was significantly altered in patients with PD. The Lanthony test is less saturated than the Farnsworth colour test, thus it is designed to detect more subtle colour deficiencies. Our patients performed worse than controls in both tests (higher C-index and S-index, reaching ranges similar to protanomalies) although these differences did not reach statistical significance as established by Bonferroni correction. Lanthony S-index $\mathrm{p}$ value was $<0.05$, indicating that our patients had a (subtle) tendency to protanomaly (S-index of 1.95).

One important limitation of this study is that only one eye was tested per person. Some recent studies suggest asymmetrical involvement of the retina in PD and recommend the incorporation of both eyes of each patient in the study. ${ }^{21}$ Thus, the diagnostic yield in this study may have been lowered by including a potentially lesser affected eye. In a similar way, including a randomly selected eye could be inappropriate for other neurological conditions, for example, a tumour compressing one optic nerve. However, incorporating both eyes of a patient may sometimes be controversial since minimum symmetric structural and functional alterations could 
Table 4 Correlation between macular and GCL structural measurements and colour vision evaluated with Lanthony colour test in patients with Parkinson's disease

\begin{tabular}{|c|c|c|c|c|c|c|}
\hline & \multicolumn{6}{|c|}{ Lanthony colour test } \\
\hline & C-index & p Value & $\mathrm{CCl}$ & p Value & S-index & p Value \\
\hline \multicolumn{7}{|l|}{ Macular thickness } \\
\hline Central & -0.019 & 0.905 & -0.059 & 0.716 & -0.017 & 0.915 \\
\hline Inner superior & -0.146 & 0.369 & -0.119 & 0.463 & -0.167 & 0.302 \\
\hline Inner nasal & -0.055 & 0.735 & -0.044 & 0.788 & -0.040 & 0.807 \\
\hline Inner inferior & -0.073 & 0.654 & -0.064 & 0.697 & -0.074 & 0.649 \\
\hline Inner temporal & -0.049 & 0.764 & -0.031 & 0.850 & -0.126 & 0.439 \\
\hline Outer superior & -0.377 & 0.017 & -0.380 & 0.015 & -0.271 & 0.090 \\
\hline Outer nasal & -0.341 & 0.031 & -0.323 & 0.042 & -0.310 & 0.051 \\
\hline Outer inferior & -0.360 & 0.022 & -0.353 & 0.025 & -0.375 & 0.017 \\
\hline Outer temporal & -0.360 & 0.023 & -0.361 & 0.022 & -0.350 & 0.027 \\
\hline Macular average & -0.691 & 0.019 & -0.657 & 0.028 & -0.709 & 0.015 \\
\hline Macular volume & -0.686 & 0.020 & -0.647 & 0.032 & -0.709 & 0.015 \\
\hline \multicolumn{7}{|l|}{ GCL thickness } \\
\hline Superior & -0.380 & 0.015 & -0.369 & 0.019 & -0.287 & 0.072 \\
\hline Superonasal & -0.383 & 0.015 & -0.337 & 0.033 & -0.350 & 0.027 \\
\hline Inferonasal & -0.338 & 0.033 & -0.313 & 0.049 & -0.268 & 0.094 \\
\hline Inferior & -0.341 & 0.031 & -0.311 & 0.051 & -0.282 & 0.078 \\
\hline Inferotemporal & -0.252 & 0.116 & -0.263 & 0.101 & -0.203 & 0.208 \\
\hline Temporal & -0.403 & 0.010 & -0.437 & 0.005 & -0.314 & 0.048 \\
\hline Average IPL+GCL & -0.381 & 0.015 & -0.358 & 0.023 & -0.319 & 0.045 \\
\hline Minimum IPL+GCL & -0.338 & 0.033 & -0.326 & 0.040 & -0.290 & 0.069 \\
\hline
\end{tabular}

have been masked and generated a per cent of dependence between measurements.

Previous studies have indicated that patients with PD lose foveal contrast sensitivity regarding patterns to which normal observers are most sensitive (ie, requiring the least contrast for detection) ${ }^{8}{ }^{9}$ Ganglion cells in the

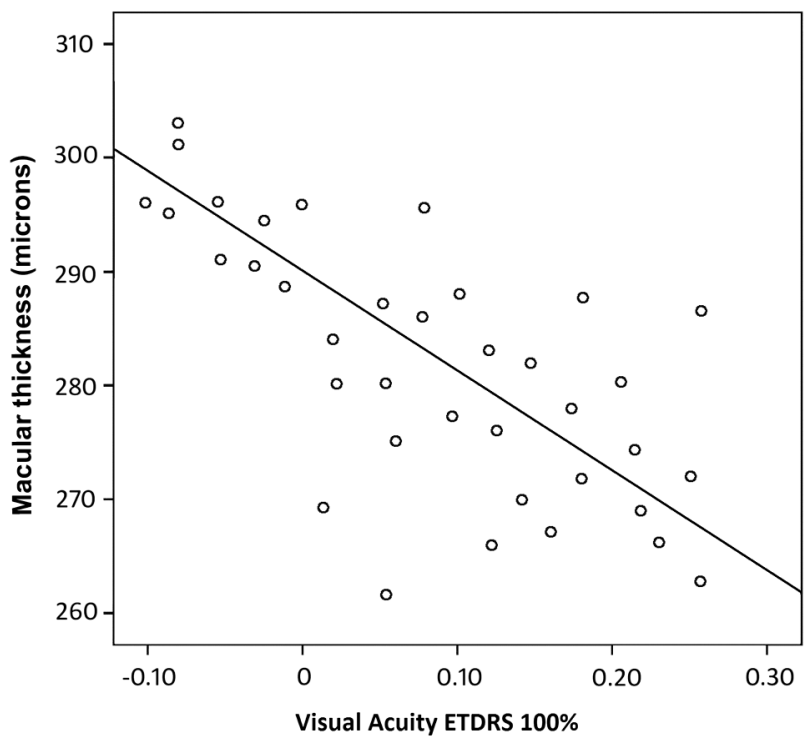

Figure 2 Correlation between the average macular thickness and visual acuity as measured with ETDRS optotype at a contrast level of $100 \%$ in patients with Parkinson's disease. ETDRS, Early Treatment Diabetic Retinopathy Study. retina show adaptation to visual contrast and pool visual inputs over their receptive fields through an array of parallel bipolar cells with smaller receptive fields. ${ }^{22}$ The parvocellular and magnocellular ganglion cells are located in the retinal ganglion cells (RGC) layer and take two different pathways for the identification of colour and

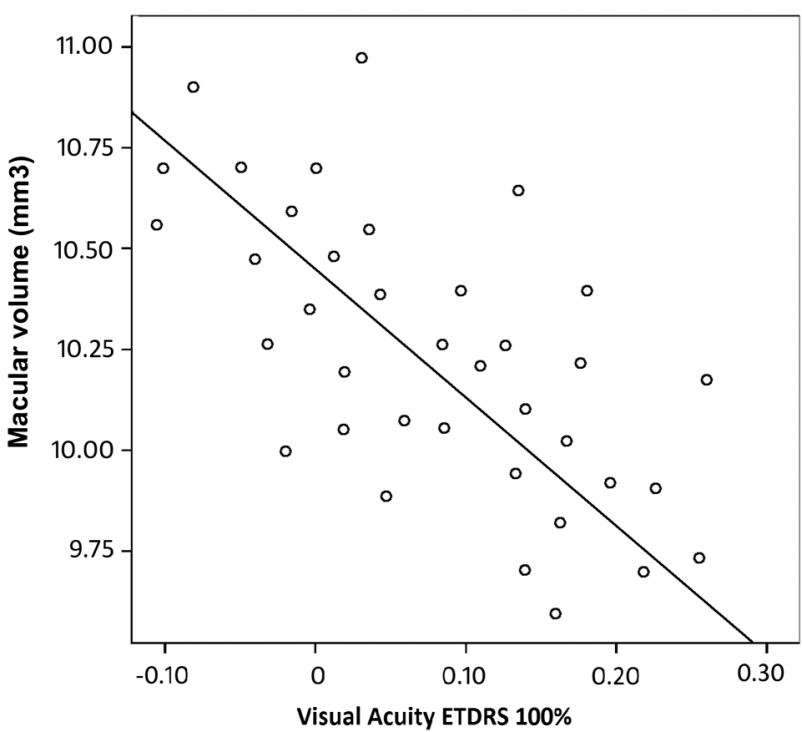

Figure 3 Correlation between macular volume and visual acuity as measured with ETDRS optotype at a contrast level of $100 \%$ in patients with Parkinson's disease. ETDRS, Early Treatment Diabetic Retinopathy Study. 
Table 5 Correlation between visual acuity measured with ETDRS chart at different levels of contrast (in \%) and macular structural measurements (thickness and volume) in patients with Parkinson's disease

\begin{tabular}{lllll} 
& Macular thickness & p Value & Macular volume & p Value \\
\hline VA ETDRS 100 & -0.765 & 0.006 & -0.761 & 0.007 \\
VA ETDRS 1.25 & -0.718 & $\mathbf{0 . 0 1 3}$ & -0.715 & $\mathbf{0 . 0 1 3}$ \\
VA ETDRS 2.50 & -0.738 & $\mathbf{0 . 0 1 0}$ & -0.729 & $\mathbf{0 . 0 1 1}$ \\
\hline
\end{tabular}

Correlation data in bold type are statistically significant $(p<0.05)$.

ETDRS, Early Treatment Diabetic Retinopathy Study; VA, visual acuity.

contrast at different frequencies. $^{23}$ RGC loss (as observed using SD-OCT) was recently identified as the cause of visual impairment in patients suffering from another neurodegenerative process, multiple sclerosis. ${ }^{24}$ Thus, a similar process could be the cause of the contrast and colour deficiencies in patients with PD. In addition, in the mammalian retina, colour vision and contrast sensitivity are modulated through D1 and D2 receptors. These dopaminergic receptors are differentially located in the retinal layers and a complete lack of activation leads to signal dispersion, and alterations in colour vision and contrast sensitivity. ${ }^{2}$

Alteration of the retinal lavers in PD was first demonstrated in 2004. ${ }^{25}$ Since then, various studies have shown different results. ${ }^{3-5}$ 25-28 Previous studies performed by our team confirmed that both macular thickness and the RNFL were affected in patients with PD, especially in the inferior and temporal quadrants. ${ }^{4}{ }^{29}$ Moreover, Garcia-Martin $e t a l^{30}$ demonstrated that the inner retinal layers were most affected in these patients, and that the GCL thickness was inversely correlated with disease duration and PD severity, and was predictive of axonal damage in these patients. The present study included a smaller number of patients, which may have affected the significance of our results compared with previous studies. We could only detect a significant reduction in the minimum GCL+IPL thickness in patients with PD compared with healthy participants, after correction for multiple comparisons. However, we detected a clear tendency towards a reduction in the macular, RNFL and GCL thicknesses. A significant reduction in the temporal sectors of the peripapillary RNFL thickness has been repeatedly observed by different groups, ${ }^{31}{ }^{32}$ and this reduction was also observed in the present study. Two recent studies, however, detected no differences in the peripapillary RNFL thickness of patients with PD compared with healthy controls, using SD-OCT, ${ }^{27} 28$ and one study only found significant differences in the nasal quadrant. ${ }^{33}$ More studies are required to clarify these contradictory observations.

In a previous study, we demonstrated that the retinal thickness corresponding to the papillomacular bundle (as measured with the Axonal Analytics software for Spectralis OCT) correlated ( $r>0.70)$ with some functional parameters (such as the mean defect and the pattern SD of the automated perimetry) in patients with PD. ${ }^{34}$ The GCL was not investigated at that time, however, and visual function parameters were reduced to perimetry and colour vision was measured with the Ishihara colour test. The current study evaluated not only the RNFL but also the GCL thickness, and more visual function parameters were analysed. The GCL correlated most with the visual function parameters: GCL thickness was directly associated with VA and CSV measured at all different spatial frequencies, and inversely correlated with the colour vision indexes. Thus, GCL thinning is linked to colour deficiencies, contrast sensitivity loss and lower vision at different contrast levels in patients with PD.

The degree of correlation is usually classified as low $(<0.30)$, moderate $(0.30-0.70)$ or strong $(>0.70)$. Our results revealed a low and moderate degree of correlation between most parameters, consistent with findings in other neurodegenerative diseases. ${ }^{35}$ Macular thickness and macular volume, however, were strongly associated with functional parameters (VA, CS, and Lanthony CCI, C-index and S-index). This strong association, to the best of our knowledge, has not been previously demonstrated in PD.

There are very few studies of the correlation between functional and structural parameters in patients with PD. Adam et $a l^{14}$ demonstrated a significant reduction in the inner retinal layer complex (RNFL+GCL+IPL) in patients with PD, but no association with contrast sensitivity (measured with the Pelli-Robson chart). A very recent study by Kaur et $a l^{15}$ demonstrated a correlation between functional parameters and GCL thinning, consistent with our results. Kaur et al, however, found no significant alterations in VA and colour vision in patients with PD, and the severity of the disease was not correlated with structural parameters, in contrast to other studies that demonstrate an association between macular and GCL thickness and disease duration and severity. ${ }^{29} 30$ Although the severity of the disease in our sample (based on the Hoehn-Yahr scale) was similar to that in Kaur's study, the duration of the disease in our study was longer than that in Kaur's study (13 vs 5 years), which may account for some of the differences in the results between the two. These discrepancies (and similarities) support the need for more studies on this topic. Our results, together with previously published studies, ${ }^{15} 30$ suggest that the GCL could be a reliable indicator of structural alterations in the retina of patients with PD, demonstrating a significant correlation with functional tests in these patients. The results of the present study 
have important implications for clinical diagnosis and functional deficits in patients with PD, and highlight the importance of visual function tests in the evaluation of these patients.

In conclusion, visual dysfunction was significantly correlated with morphological parameters in patients with PD. Patients with PD present with a reduction in GCL thickness, which is closely associated with visual dysfunction.

Contributors VP contributed to research project: organisation; statistical analysis and manuscript: review and critique. MS contributed to research project: conception, design, organisation and execution; statistical analysis: review and critique; manuscript: writing of the first draft, review and critique. MJR contributed to research project: organisation and execution; statistical analysis: review and critique; manuscript: writing of the first draft, review and critique. SO and RA contributed to research project: organisation and execution; statistical analysis: review and critique; manuscript: review and critique. MPB and MIF contributed to research project: execution; statistical analysis: review and critique; manuscript: review and critique. JML and LEP contributed to research project: execution; statistical analysis: review and critique; manuscript: review and critique. EG-M contributed to research project: conception, design, organisation and execution; statistical analysis: design, execution, review and critique; manuscript: writing of the first draft, review and critique.

Funding This research received no specific grant from any funding agency in the public, commercial or not-for-profit sectors.

Competing interests None declared.

Patient consent Obtained.

Ethics approval Comité Etico de Investigación Científica de Aragón (CEICA)

Provenance and peer review Not commissioned; externally peer reviewed.

Data sharing statement No additional data are available.

Open Access This is an Open Access article distributed in accordance with the Creative Commons Attribution Non Commercial (CC BY-NC 4.0) license, which permits others to distribute, remix, adapt, build upon this work noncommercially, and license their derivative works on different terms, provided the original work is properly cited and the use is non-commercial. See: http:// creativecommons.org/licenses/by-nc/4.0/

\section{REFERENCES}

1. Bodis-Wollner I. Retinopathy in Parkinson disease. J Neural Transm (Vienna) 2009;116:1493-501.

2. Hajee ME, March WF, Lazzaro DR, et al. Inner retinal layer thinning in Parkinson disease. Arch Ophthalmol 2009;127:737-41.

3. Cubo E, Tedejo RP, Rodriguez Mendez V. Retina thickness in Parkinson's disease and essential tremor. Mov Disord 2010;25: 2461-77.

4. Satue M, Garcia-Martin E, Fuertes I, et al. Use of Fourier-domain OCT to detect retinal nerve fiber layer degeneration in Parkinson's disease patients. Eye (Lond) 2013;27:507-14.

5. Garcia-Martin E, Satue M, Fuertes I, et al. Ability and reproducibility of Fourier domain optical coherence tomography to detect retinal nerve fiber layer atrophy in Parkinson's disease. Ophthalmology 2012;119:2161-7.

6. Fisher JB, Jacobs DA, Markowitz CE, et al. Relation of visual function to retinal nerve fiber layer thickness in multiple sclerosis. Ophthalmology 2006;113:324.

7. Parisi V, Manni G, Spadaro M, et al. Correlation between morphological and functional retinal impairment in multiple sclerosis patients. Invest Ophthalmol Vis Sci 1999;40:2520-7.

8. Bodis-Wollner I. Visual acuity and contrast sensitivity in patients with cerebral lesions. Science 1972:178:769-71.
9. Bodis-Wollner I, Diamond S. The measurement of spatial contrast sensitivity in cases of blurred vision associated with cerebral lesions. Brain 1976;99:695-710.

10. Price MJ, Feldman RG, Adelberg D, et al. Abnormalities in color vision and contrast sensitivity in Parkinson's disease. Neurology 1992;42:887-90.

11. Oh YS, Kim JS, Chung SW et al. Color vision in Parkinson's disease and essential tremor. Eur J Neurol 2011;18:577-83.

12. Hipp G, Diedericha NJ, Pieria V, et al. Primary vision and facial emotion recognition in early Parkinson's disease. J Neurol Sci 2014;338:178-82.

13. Archibald NK, Clarke MP, Mosimann UP, et al. Retinal thickness in Parkinson's disease. Parkinsonism Relat Disord 2011;17:431-6.

14. Adam CR, Shrier E, Ding Y, et al. Correlation of inner retinal thickness evaluated by spectral-domain optical coherence tomography and contrast sensitivity in Parkinson disease. J Neuroophthalmol 2013;33:137-42.

15. Kaur M, Saxena R, Singh D, et al. Correlation between structural and functional retinal changes in Parkinson disease. J Neuroophthalmol 2015;35:254-8.

16. Gelb DJ, Oliver E, Gilman S. Diagnostic criteria for Parkinson disease. Arch Neurol 1999;56:33-9.

17. Hoehn MM, Yahr MD. Parkinsonism: onset, progression and mortality. Neurology 1967;17:427-42.

18. Ramaker C, Marinus J, Stiggelbout AM, et al. Systematic evaluation of rating scales for impairment and disability in Parkinson's disease. Mov Disord 2002;17:867-76.

19. Vingrys AJ, King-Smith PE. A quantitative scoring technique for panel tests of color vision. Invest Ophthalmol Vis Sci 1988;29:50-63.

20. Bowman AJ. A method for quantitative scoring of the Farnsworth panel D15. Acta Ophthalmol (Copenh) 1982;60:907-16.

21. Shrier EM, Adam CR, Spund B, et al. Interocular asymmetry of foveal thickness in Parkinson disease. J Ophthalmol 2012;2012:728457.

22. Kim KJ, Rieke F. Temporal contrast adaptation in the input and output signals of salamander retinal ganglion cells. J Neurosci 2001;21:287-99.

23. Laycock R, Crewther SG, Crewther DP. A role for the 'magnocellular advantage' in visual impairments in neurodevelopmental and psychiatric disorders. Neurosci Biobehav Rev 2007;31:363-76.

24. Lampert EJ, Andorra M, Torres-Torres R, et al. Color vision impairment in multiple sclerosis points to retinal ganglion cell damage. J Neurol 2015;262:2491-7.

25. Inzelberg R, Ramirez JA, Nisipeanu $P$, et al. Retinal nerve fiber layer thinning in Parkinson's disease. Vision Res 2004;44:2793-7.

26. Altintaș $\mathrm{O}$, Ișeri $\mathrm{P}, \mathrm{Ozk}$ an $\mathrm{B}$, et al. Correlation between retinal morphological and functional findings and clinical severity in Parkinson's disease. Doc Ophthalmol 2008;116:137-46.

27. Bittersohl D, Stemplewitz B, Keserü M et al. Detection of retinal changes in idiopathic Parkinson's disease using high-resolution optical coherence tomography and Heidelberg retina tomography. Acta Ophthalmol 2015;93:e578-84.

28. Chorostecki J, Seraji-Bozorgzad N, Shah A, et al. Characterization of retinal architecture in Parkinson's disease. J Neurol Sci 2015;355:44-8.

29. Satue M, Seral M, Otin S, et al. Retinal thinning and correlation with functional disability in patients with Parkinson's disease. $\mathrm{Br} \mathrm{J}$ Ophthalmol 2014;98:350-5.

30. Garcia-Martin E, Larrosa JM, Polo V, et al. Distribution of retinal layer atrophy in patients with Parkinson disease and association with disease severity and duration. Am J Ophthalmol 2014;157: 470-8.

31. Sari ES, Koc R, Yazici A, et al. Ganglion cell-inner plexiform layer thickness in patients with Parkinson disease and association with disease severity and duration. J Neuroophthalmol 2015;35:117-21.

32. La Morgia C, Barboni P, Rizzo G, et al. Loss of temporal retinal nerve fibers in Parkinson disease: a mitochondrial pattern? Eur J Neurol 2013;20:198-201.

33. Bayhan HA, Aslan Bayhan S, Tanık N, et al. The association of spectral-domain optical coherence tomography determined ganglion cell complex parameters and disease severity in Parkinson's disease. Curr Eye Res 2014;39:1117-22.

34. Satue M, Bambo M, Garcia-Martin E, et al. Correlation between function and structure of retinal nerve fiber layer in Parkinson disease. Acta Ophthalmol 2012;90

35. Garcia-Martin E, Rodriguez-Mena D, Herrero R, et al. Neuro-ophthalmologic evaluation, quality of life and functional disability in MS patients. Neurology 2013;81:1-8. 